\title{
Improving fixed rate insulin infusion prescriptions in patients admitted with diabetic ketoacidosis in Basildon Hospital - a local quality improvement project
}

\author{
Authors: Uwais Dudhiya, ${ }^{\mathrm{A}}$ Lagzouli Nora, ${ }^{\mathrm{A}}$ Doreen Ko, ${ }^{\mathrm{A}}$ Karolina Kapellar ${ }^{\mathrm{B}}$ and Alan Choo-kang ${ }^{\mathrm{A}}$
}

\begin{abstract}
Background
Diabetic ketoacidosis (DKA) is a life-threatening complication of type 1 diabetes and some cases of type 2 diabetes. Intravenous fluids and fixed rate insulin infusion (FRII) remain the mainstay of treatment for this condition. ${ }^{1}$ However, given the complex nature of the treatment regime of this serious condition, there is considerable variation on how treatment is prescribed for these patients. Our quality improvement project aimed to improve the number of correctly prescribed FRII at Basildon Hospital over the course of 14 months.
\end{abstract}

\section{Aims}

> To determine the number of patients admitted into Basildon Hospital with diabetic ketoacidosis.

> To investigate the proportion of correctly prescribed fixed rate insulin in patients admitted with diabetic ketoacidosis.

$>$ To introduce interventions in the form of a quality improvement project to improve the proportion of correctly prescribed fixed rate insulin infusion prescriptions.

\section{Methods}

A prospective database of patients admitted under the medical team at Basildon Hospital was used to identify patients who were admitted with DKA between April 2017 and May 2018. We subsequently retrieved and analysed the prescription charts of these patients and recorded whether the patient had fixed rate insulin prescribed correctly. After recognising the need for interventions to improve the results, we planned to improve education for doctors with regards to the prescribing of correct treatment in the form of emails and education posters.

\section{Results}

Our aim was to have $100 \%$ correctly prescribed insulin prescriptions for patients who were admitted with DKA. Prior to us starting our quality improvement project, the percentage of correctly prescribed insulin in April 2017 was $12.5 \%$. We then sent an email to our colleagues in Basildon Hospital as our first intervention. The email was sent to all doctors, including those who are involved in seeing patients on admission. This initially improved the percentage to $100 \%$ over the coming month; however, subsequently the percentage of correctly prescribed insulin dropped significantly to $60 \%$. Over the next few months our target was not reached so we sent a repeat email to our colleagues in October 2017. This initially had our desired effect; however, the percentage dropped again after November 2017. We then displayed a poster within the acute medical unit to attempt to improve our rates, unfortunately this did not improve them to the desired level.

\section{Conclusion}

We recognise the need for continuous education to ensure the percentage of correctly prescribed FRII remains satisfactory. Our aim in the future is to introduce a local formal proforma, which allows for easy prescription of FRII and avoid prescribing errors.

\section{Reference}

1 Joint British Diabetes Societies Inpatient Care Group. The management of diabetic ketoacidosis in adults. Joint British Diabetes Societies Inpatient Care Group, 2013. www.diabetes.org.uk/professionals/position-statements-reports/specialist-care-for-children-andadults-and-complications/the-management-of-diabetic-ketoacidosis-in-adults [Accessed: 3 November 2018].

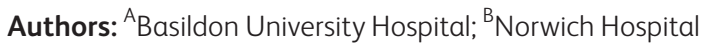

